Yukawa Institute Kyoto

quant-ph/9611003

February 1996

\title{
Generally Deformed Oscillator, Isospectral Oscillator System and Hermitian Phase Operator
}

\author{
Hong-Chen Fu円 and Ryu Sasaki角 \\ Yukawa Institute for Theoretical Physics, Kyoto University \\ Kyoto 606-01, Japan
}

\begin{abstract}
The generally deformed oscillator (GDO) and its multiphoton realization as well as the coherent and squeezed vacuum states are studied. We discuss, in particular, the GDO depending on a complex parameter $q$ (therefore we call it $q$-GDO) together with the finite dimensional cyclic representations. As a realistic physical system of GDO the isospectral oscillator system is studied and it is found that its coherent and squeezed vacuum states are closely related to those of the oscillator. It is pointed out that starting from the $q$-GDO with $q$ root of unity one can define the hermitian phase operators in quantum optics consistently and algebraically. The new creation and annihilation operators of the Pegg-Barnett type phase operator theory are defined by using the cyclic representations and these operators degenerate to those of the ordinary oscillator in the classical limit $q \rightarrow 1$.
\end{abstract}

PACS numbers: 03.65.-w, 02.20.-a, 42.50.-p

Journal of Physics A: Mathematical and General

29 (1996) 4049

\footnotetext{
${ }^{1}$ JSPS Fellow. On leave of absence from Institute of Theoretical Physics, Northeast Normal University, Changchun 130024, P.R.China. E-mail: hcfu@yukawa.kyoto-u.ac.jp

${ }^{2}$ Supported partially by the grant-in-aid for Scientific Research, Priority Area 231 "Infinite Analysis" and General Research (C) in Physics, Japan Ministry of Education.
} 


\section{Introduction}

Deformation of Lie algebras has been finding applications in various branches of physics. The $q$-deformed Lie algebras, or the quantum algebras, play an important role in quantum integrable models and quantum inverse scattering method [1, 2]. The generally deformed oscillator (GDO) first appeared in Heisenberg's theory of nonlinear spinor dynamics [3]. In the literature, many more deformed oscillators can be found [4, 5, 6, 7] and a unification scheme for them has been suggested (see [8] and references therein). Many physical systems are found to enjoy the GDO symmetry (for a list see [8, 9, 10]). In this paper we shall pay attention to several types of GDO, namely the multiphoton realization of GDO and two new GDO systems: the isospectral oscillator system (ISOS) [11, 12] and $q$-deformed GDO which is a subalgebra of GDO. Based on the $q$-deformed GDO having finite dimensional cyclic representations we construct the hermitian phase operator in quantum optics algebraically.

In Sec. 2 we first review the GDO and study its multiphoton realization and the coherent and squeezed vacuum states. These states are expressed in terms of an exponential displacement operator acting on the vacuum state. We know that the GDO can be realized in terms of the usual single photon operator [8, 13] (multiphoton realization of some Lie algebras and the $q$-oscillator can be found in [14] and [15, 16, 17], respectively, and the single photon realization of $q$-oscillator has been extensively studied [18]), and that the exponential coherent states of the GDO are already obtained in a different way 13. A new notion of 'spontaneously broken' multiphoton realization of $q$-oscillators is introduced here.

In Sec.3 we add a new member to the GDO family, the isospectral oscillator system (ISOS) 11. As suggested by the name, it has the same spectrum as the oscillator. It can be formulated in the framework of supersymmetric quantum mechanics and factorization method [12]. Its coherent states are studied in [19]. We show that the creation and annihilation operators and the Hamiltonian generate a GDO. We also study its coherent and squeezed vacuum states and find that these states are closely related to the density-dependent annihilation operator coherent states and to the squeezed vacuum of the oscillator, respectively.

Section 4 is devoted to the connection between the hermitian phase operator in quantum optics and the $q$-GDO with cyclic representations. The proper quantization of the phase angle of an oscillator was first considered by Dirac in 1927 [20]. For history and some review papers of the phase operator, see [21]. However, because of the fact that the creation and

annihilation operators of the oscillator do not admit a naive polar decomposition, ie. a product of a unitary times a positive semi-definite hermitian operator, the problem kept unsolved for a long time. Susskind and Glogower considered a weaker exponential phase 
operator which is one-side unitary [22], namely only one of the two relations $U U^{\dagger}=1$ or $U^{\dagger} U=1$ holds for the exponential phase operator $U=e^{i \phi}$. Recently it was realized that the hermitian phase operator could be defined in an $(S+1)$-dimensional Hilbert space and that the expectation values of physical quantities would tend to those of the oscillator in the limit $S \rightarrow \infty$ [23, 24]. Pegg and Barnett considered a truncated oscillator defined in the $(S+1)$-dimensional space and presented the hermitian phase operator (PB phase operator) 23. The truncated oscillator has one disadvantage from the symmetry point of view: its operators do not form a closed algebra. Moreover, the truncated oscillator is not the only way to realize the PB phase operator. For example, Ellinas revealed the relevance of the PB phase operator to the naive $q$-oscillator [6] with $q$ root of unity [25]. In his approach, however, the the naive $q$-oscillator provides only a finite dimensional space and the hermiticity of the phase operator is not automatically ensured because of the use of a regular representation (see Sec.4). In order to connect GDO with the hermitian phase operator, we introduce a new GDO, which is a subalgebra of GDO depending on a complex parameter $q$ (therefor we call it $q$-GDO). It has finite dimensional cyclic representations when $q$ is a root of unity. We here advance the problem in two points: (1) the $q$-GDO with $q$ root of unity is particularly suited for algebraic realization of the PB phase operator theory; and (2) the cyclic representation of $q$-GDO (and therefore the ' $q$-oscillator') ensures automatically the hermiticity of the phase operator.

\section{Generally Deformed Oscillator}

In this section we first review the GDO, then the coherent and the squeezed vacuum states of GDO are presented explicitly in terms of an ordinary exponential operator.

\subsection{GDO and its multiphoton realization}

The GDO is an associative algebra $\mathcal{B}$ over the complex number field $\mathrm{C}$ with generators $A^{\dagger}, A, \mathcal{N}$ and the unit 1 satisfying

$$
\left[\mathcal{N}, A^{\dagger}\right]=A^{\dagger}, \quad[\mathcal{N}, A]=-A, \quad A A^{\dagger}=F(\mathcal{N}+1), \quad A^{\dagger} A=F(\mathcal{N})
$$

where the hermitian non-negative function $F$ is called the structure function. It should satisfy the condition

$$
F(0)=0
$$

in order to have the Fock representation. 
The algebra (2.1) can be realized in terms of the usual single photon operator [8, 13] (multiphoton realization of some Lie algebras and the $q$-oscillator can be found in [14] and [15, 16, 17], respectively, and the single photon realization of $q$-oscillator has been extensively studied [18]). Here we would like to present the general multiphoton realization of GDO. For this purpose, we consider the multiphoton lowering operator

$$
A=f(N) a^{m}
$$

where $a$ and $a^{\dagger}$ are the annihilation and creation operators of the photon satisfying $\left[a, a^{\dagger}\right]=$ 1, $N=a^{\dagger} a$, and $m$ is a positive integer. As usual the Fock states of the oscillator $a$ and $a^{\dagger}$ are denoted by $|n\rangle, n=0,1, \ldots ; a|0\rangle=0, a|n\rangle=\sqrt{n}|n-1\rangle, a^{\dagger}|n\rangle=\sqrt{n+1}|n+1\rangle$. The function $f(N)$ specifies the intensity dependent coupling, which is in general complex and we assume that $f(x)$ does not have zeros at non-negative integer values of $x$. By using $a a^{\dagger}=N+1, a^{2}\left(a^{\dagger}\right)^{2}=(N+1)(N+2)$, etc, we obtain

$$
\begin{aligned}
& A A^{\dagger}=(N+1)(N+2) \cdots(N+m) f(N) f^{*}(N), \\
& A^{\dagger} A=(N-m+1)(N-m+2) \cdots N f(N-m) f^{*}(N-m) .
\end{aligned}
$$

It is obvious that we only need to restrict our discussion to the sector $S_{i}(i=0,1, \cdots, m-1)$ spanned by the Fock states $|n m+i\rangle$ ( $n$ non-negative integers). Introducing the multiphoton number operator $\mathcal{N}_{i} \equiv \mathcal{N}(i=0,1, \cdots, m-1)$ on the sector $S_{i}$

$$
\mathcal{N}=\frac{1}{m}(N-i), \quad i=0,1, \cdots, m-1,
$$

and $F(\mathcal{N}+1) \equiv(m \mathcal{N}+1+i) \cdots(m \mathcal{N}+m+i) f(m \mathcal{N}+i) f^{*}(m \mathcal{N}+i)$, we can recast the system (2.4), (2.5) in the following form

$$
A A^{\dagger}=F(\mathcal{N}+1), \quad A^{\dagger} A=F(\mathcal{N}), \quad\left[\mathcal{N}, A^{\dagger}\right]=A^{\dagger}, \quad[\mathcal{N}, A]=-A,
$$

which we call intensity-dependent m-photon realization of $\mathcal{B}$. Note that the r.h.s. of (2.5) vanishes on the Fock states $|n\rangle$ for $0 \leq n \leq m-1$, which implies $F(0)=0$ in each sector.

It is very tempting to apply the idea of the system (2.7) to the multiphoton ( $m$-photon) realization of the $q$-deformed oscillator. Let us choose (here $q$ is a real deformation parameter)

$$
f(N) \equiv\left\{\frac{1}{(N+1) \cdots(N+m)}\left[\frac{N}{m}+1\right]\right\}^{\frac{1}{2}}
$$

where $[x] \equiv\left(q^{x}-q^{-x}\right) /\left(q-q^{-1}\right)$, and define

$$
b_{q} \equiv A=f(N) a^{m}, \quad b_{q}^{\dagger} \equiv A^{\dagger}=\left(a^{\dagger}\right)^{m} f^{*}(N), \quad N_{q} \equiv \mathcal{N}+\frac{i}{m} .
$$


Then by using (2.4) and (2.5) we would obtain formally the following relations

$$
\begin{aligned}
& b_{q} b_{q}^{\dagger}=\left[N_{q}+1\right], \quad\left[N_{q}, b_{q}^{\dagger}\right]=b_{q}^{\dagger}, \quad\left[N_{q}, b_{q}\right]=-b_{q} . \\
& b_{q}^{\dagger} b_{q}=\frac{(N-m+1)(N-m+2) \cdots N}{(N-m+1)(N-m+2) \cdots N}\left[N_{q}\right]=\left[N_{q}\right] .
\end{aligned}
$$

Eqs. 2.10), 2.11) are in fact a multiphoton realization of the $q$-oscillator,

$$
b_{q} b_{q}^{\dagger}-q b_{q}^{\dagger} b_{q}=q^{-N_{q}}
$$

It should be remarked that the eigenvalues of $N_{q}$ are not integers except for the $i=0$ sector.

By close inspection, however, one finds that the relation (2.11) is not true in the "vacuum" of each sector $S_{i}\left(i \geq 1\right.$ and $m>1$ ) (for the $m=1$ case see [18, the $S_{0}$ sector is discussed in [15]). Obviously the "vacuum" of the $i$-th sector $\| 0\rangle=|i\rangle$ vanishes when applied by $b_{q}$,

$$
\left.b_{q} \| 0\right\rangle=f(N) a^{m}|i\rangle=0, \quad i=0,1, \ldots, m-1 .
$$

On the other hand, as remarked above, $\left.\left.\left[N_{q}\right] \| 0\right\rangle=\left[\frac{i}{m}\right] \| 0\right\rangle$ is non-vanishing for $i \geq 1$. This apparent inconsistency is caused by $0 / 0=1$ in (2.11), since $N-i$ in the numerator and denominator vanish on $\| 0\rangle=|i\rangle$. To sum up, the relations (2.11)and (2.12) are broken only by the "vacuum" expectation value and all the other relations are correct. It would be very interesting if one could find physical applications of the 'spontaneously broken' multiphoton realization of $q$-oscillator.

If we introduce the intensity and sector-dependent multiphoton coupling then we can obtain the $q$-oscillator in each sector. Namely, if we define

$$
a_{q}=\sqrt{\frac{[\mathcal{N}+1]}{(N+1) \cdots(N+m)}} a^{m}
$$

in each sector, then it is easy to see that $a_{q} a_{q}^{\dagger}=[\mathcal{N}+1]$ and $a_{q}^{\dagger} a_{q}=[\mathcal{N}]$ are satisfied as operator equations. This result has been reported in [16] but they considered this realization only in the sector $S_{0}$.

\subsection{Coherent and squeezed vacuum states}

In this subsection we shall study the ladder-operator coherent and squeezed vacuum states of the GDO. To this end we define a convenient orthonormal basis for $S_{i}$

$$
\left.\| n\rangle=\frac{1}{\sqrt{\llbracket F(n) \rrbracket !}}\left(A^{\dagger}\right)^{n} \| 0\right\rangle,
$$

where $\| 0\rangle=|i\rangle$ is the vacuum state of the sector $S_{i}$ satisfying $\left.\left.A \| 0\right\rangle=\mathcal{N} \| 0\right\rangle=0$ and $\llbracket F(n) \rrbracket ! \equiv F(n) F(n-1) \cdots F(1), \quad \llbracket F(0) \rrbracket ! \equiv 1$. On this basis we have

$$
\left.\left.\left.\left.\left.\left.A^{\dagger} \| n\right\rangle=\sqrt{F(n+1)} \| n+1\right\rangle, \quad A \| n\right\rangle=\sqrt{F(n)} \| n-1\right\rangle, \quad \mathcal{N} \| n\right\rangle=n \| n\right\rangle .
$$




\subsubsection{Squeezed vacuum and squeeze operator}

We first consider the squeezed vacuum $|v\rangle$ annihilated by $\mu A+\nu A^{\dagger}$

$$
\left(\mu A+\nu A^{\dagger}\right)|v\rangle=0
$$

where the complex numbers $\mu$ and $\nu$ satisfy $|\nu / \mu|<1$. Let us express it in the form of an exponential displacement-operator (squeeze operator) acting on the vacuum state. Expand $\left.|v\rangle=\sum_{n=0}^{\infty} C_{n} \| n\right\rangle$ and insert it into (2.16), yielding

$$
C_{2 k+1}=0, \quad C_{2 k}=C_{0} z^{k} \sqrt{\frac{\llbracket F(2 k-1) \rrbracket ! !}{\llbracket F(2 k) \rrbracket ! !}},
$$

where $z=-\nu / \mu, \llbracket F(2 k) \rrbracket ! !=F(2 k) F(2 k-2) \cdots F(2), \llbracket F(2 k-1) \rrbracket ! !=F(2 k-1) F(2 k-$ $3) \cdots F(1)$ and $\llbracket F(0) \rrbracket ! !=\llbracket F(-1) \rrbracket ! ! \equiv 1$. Then we have

$$
\left.\left.|v\rangle=C_{0} \sum_{k=0}^{\infty} z^{k} \sqrt{\frac{\llbracket F(2 k-1) \rrbracket ! !}{\llbracket F(2 k) \rrbracket ! !}} \| 2 k\right\rangle=C_{0} \sum_{k=0}^{\infty} z^{k} \frac{\left(A^{\dagger 2}\right)^{k}}{\llbracket F(2 k) \rrbracket ! !} \| 0\right\rangle .
$$

It is easy to check that the above infinite series converges if $|z|<1$ under mild assumptions on the asymptotic behavior of $f(x)$, e.g., $f(x) \simeq x^{\alpha}$ for $x \rightarrow \infty$. Now, as a key step, we use the following identity

$$
\left(\frac{\mathcal{N}}{F(\mathcal{N})} A^{\dagger 2}\right)^{k}=\left(A^{\dagger 2}\right)^{k} \frac{\mathcal{N}+2}{F(\mathcal{N}+2)} \cdots \frac{\mathcal{N}+2 k}{F(\mathcal{N}+2 k)}
$$

which, on the vacuum state $\| 0\rangle$, becomes

$$
\left.\left.\left.\left(\frac{\mathcal{N}}{F(\mathcal{N})} A^{\dagger 2}\right)^{k} \| 0\right\rangle=\left(A^{\dagger 2}\right)^{k} \frac{\llbracket(2 k) \rrbracket ! !}{\llbracket F(2 k) \rrbracket ! !} \| 0\right\rangle=\left(A^{\dagger 2}\right)^{k} \frac{2^{k} k !}{\llbracket F(2 k) \rrbracket ! !} \| 0\right\rangle .
$$

Then we can rewrite 2.18) as

$$
\left.\left.|v\rangle=C_{0} \sum_{k=0}^{\infty} \frac{1}{k !}\left(\frac{z}{2} A^{\dagger 2}\right)^{k} \frac{(\mathcal{N}+2) \cdots(\mathcal{N}+2 k)}{F(\mathcal{N}+2) \cdots F(\mathcal{N}+2 k)} \| 0\right\rangle=C_{0} \exp \left(\frac{z \mathcal{N}}{2 F(\mathcal{N})} A^{\dagger 2}\right) \| 0\right\rangle
$$

Following the terminology of the oscillator, the operator

$$
S(z)=C_{0} \exp \left(\frac{z \mathcal{N}}{2 F(\mathcal{N})} A^{\dagger 2}\right)
$$

is referred to as the generalized squeeze operator. 


\subsubsection{Multiphoton coherent states}

The multiphoton coherent states are the eigenstate of the annihilation operator $A$

$$
A|\alpha\rangle=\alpha|\alpha\rangle,
$$

where $\alpha$ is an arbitrary complex number. Expanding $\left.|\alpha\rangle=\sum_{n=0}^{\infty} D_{n} \| n\right\rangle$ and inserting it into (2.23), we have $D_{n}=D_{0} \alpha^{n} / \sqrt{\llbracket F(n) \rrbracket !}$ and the coherent state $|\alpha\rangle$ is obtained as

$$
\left.|\alpha\rangle=D_{0} \sum_{n=0}^{\infty} \frac{\alpha^{n}}{\llbracket F(n) \rrbracket !}\left(A^{\dagger}\right)^{n} \| 0\right\rangle .
$$

Using the following identity

$$
\left(\frac{\mathcal{N}}{F(\mathcal{N})} A^{\dagger}\right)^{n}=\left(A^{\dagger}\right)^{n} \frac{\mathcal{N}+1}{F(\mathcal{N}+1)} \cdots \frac{\mathcal{N}+n}{F(\mathcal{N}+n)}
$$

we obtain the coherent state in the ordinary exponential form

$$
\left.\left.\left.|\alpha\rangle=C_{0} \sum_{n=0}^{\infty} \frac{\alpha^{n}}{n !}\left(\frac{\mathcal{N}}{F(\mathcal{N})} A^{\dagger}\right)^{n} \| 0\right\rangle=C_{0} \exp \left(\frac{\alpha \mathcal{N}}{F(\mathcal{N})} A^{\dagger}\right) \| 0\right\rangle=C_{0} D(\alpha) \| 0\right\rangle .
$$

We remark that the coherent states of GDO have already been studied extensively. In particular, Shanta et. al. have obtained the result (2.26) [13 using a different method (for the $q$-oscillator case see [29]). They first look for an operator $G^{\dagger}$ such that $\left[A, G^{\dagger}\right]=1$ and then write the displacement operator as $D(\alpha)=\exp \left(\alpha G^{\dagger}\right)$. In fact, by direct verification, we have

$$
\left[A, \frac{\mathcal{N}}{F(\mathcal{N})} A^{\dagger}\right]=1
$$

Our method is using the identity (2.19) and (2.25), by which we can easily obtain not only the coherent states but also the squeezed vacuum states. Furthermore, we shall find that this method will play an important role in revealing the relevance of the coherent and squeezed vacuum states of ISOS to those of the oscillator (see Sec.3.2).

In some works the so-called deformed exponential displacement operator $\exp _{F}\left(\alpha A^{\dagger}\right) \equiv$ $\sum_{n=0}^{\infty} \frac{\alpha^{n}\left(A^{\dagger}\right)^{n}}{\llbracket F(n) \rrbracket !}$ is used to express the coherent state $\left.\exp _{F}\left(\alpha A^{\dagger}\right) \| 0\right\rangle$. We note that two displacement operators $\exp _{F}\left(\alpha A^{\dagger}\right)$ and $\exp \left(\frac{\alpha \mathcal{N}}{F(\mathcal{N})} A^{\dagger}\right)$ are essentially different, although they give rise to the same coherent states by acting on the vacuum state.

From the above discussion we see that the $q$-oscillator also admits the multi-component squeezed and coherent states through its multiphoton realization (2.9) but the relationship is broken by the "vacuum" expectation value except for in $S_{0}$. 


\section{GDO and Isospectral oscillator system}

\subsection{Isospectral oscillator system as a GDO}

In this section we shall first review some basic facts of ISOS and then show that its creation and annihilation operators and the Hamiltonian generate a GDO. The ISOS, as suggested by the name, is a system having the same spectrum as the ordinary oscillator. The Hamiltonian of the oscillator is $H=a^{\dagger} a+\frac{1}{2}=N+\frac{1}{2}$. Then the ISOS Hamiltonian is

$$
H_{\lambda}=b^{\dagger} b+\frac{1}{2}=N_{\lambda}+\frac{1}{2}
$$

where $b$ and its conjugate $b^{\dagger}$ is defined by

$$
b b^{\dagger}=a a^{\dagger},
$$

and $N_{\lambda}=b^{\dagger} b$. For the realization of the operators $b^{\dagger}$ and $b$ in the coordinate representation, see [11, 12]. In fact, $\lambda$ is just a parameter entering the coordinate representation of $b^{\dagger}$ and b. From the relation

$$
H_{\lambda} b^{\dagger}=b^{\dagger}(H+1)
$$

it follows that the states $(|n-1\rangle$ are the eigenstates of $H)$

$$
\left|\psi_{n}\right\rangle=\frac{1}{\sqrt{n}} b^{\dagger}|n-1\rangle, \quad n=1,2, \cdots
$$

are the normalized orthogonal eigenstates of $H_{\lambda}$ with eigenvalues $E_{n}=n+\frac{1}{2}$. These states, together with the state $\left|\psi_{0}\right\rangle$ which is defined by $b\left|\psi_{0}\right\rangle=0$ and is an eigenstate of $H_{\lambda}$ with eigenvalue $1 / 2$, are complete. The operators $b^{\dagger}$ and $b$ transform the eigenstates of $H_{\lambda}$ to those of $H$ and vice versa,

$$
b^{\dagger}|n\rangle=\sqrt{n+1}\left|\psi_{n+1}\right\rangle, \quad b\left|\psi_{n}\right\rangle=\sqrt{n}|n-1\rangle .
$$

The creation and annihilation operators of ISOS are found to be [11, 12]

$$
A=b^{\dagger} a b, \quad A^{\dagger}=b^{\dagger} a^{\dagger} b
$$

The operators $A$ and $A^{\dagger}$ do not give a closed (Lie) algebra as argued in [19. Here we are interested in the associative algebra generated by $A^{\dagger}, A$ and $N_{\lambda}$ (or $H_{\lambda}$ ). From the above relations, it is not difficult to derive

$$
\left[N_{\lambda}, A^{\dagger}\right]=A^{\dagger}, \quad\left[N_{\lambda}, A\right]=-A, \quad A^{\dagger} A=\left(N_{\lambda}-1\right)^{2} N_{\lambda}, \quad A A^{\dagger}=N_{\lambda}^{2}\left(N_{\lambda}+1\right),
$$


which is just a GDO with the structure function

$$
F(x)=(x-1)^{2} x
$$

We denote this algebra by $\mathcal{B}_{\lambda}$. It is easy to prove that the algebra $\mathcal{B}_{\lambda}$ has two orthogonal vacuum states $\left|\psi_{0}\right\rangle$ and $\left|\psi_{1}\right\rangle$ defined by $A\left|\psi_{0}\right\rangle=A\left|\psi_{1}\right\rangle=0$, which correspond to the two zeroes of $F(x)$. The one-dimensional subspace $\left\{\left|\psi_{0}\right\rangle\right\}$ is invariant and it forms a one-dimensional representation of $\mathcal{B}_{\lambda}$

$$
A\left|\psi_{0}\right\rangle=A^{\dagger}\left|\psi_{0}\right\rangle=N_{\lambda}\left|\psi_{0}\right\rangle=0, \quad H_{\lambda}\left|\psi_{0}\right\rangle=\frac{1}{2}\left|\psi_{0}\right\rangle
$$

The subspace spanned by $\left\{\left|\psi_{n}\right\rangle \mid n=1,2, \cdots,\right\}$ is also an invariant space on which the representation can be easily obtained as

$$
A\left|\psi_{n}\right\rangle=(n-1) \sqrt{n}\left|\psi_{n-1}\right\rangle, \quad A^{\dagger}\left|\psi_{n}\right\rangle=n \sqrt{n+1}\left|\psi_{n+1}\right\rangle, \quad H_{\lambda}\left|\psi_{n}\right\rangle=\left(n+\frac{1}{2}\right)\left|\psi_{n}\right\rangle .
$$

The representation (3.10) is an infinite dimensional irreducible representation. Therefore the whole Hilbert space is decomposed into a direct sum of two irreducible subspaces.

\subsection{Coherent state and squeezed vacuum of ISOS}

We now turn to the coherent and the squeezed vacuum states of the ISOS, with special emphasis on their relationship with those of the oscillator. The coherent states of ISOS as the eigenstates of $A$ with the eigenvalue $\alpha$ has already been obtained as 19

$$
|\alpha\rangle=\frac{1}{\sqrt{{ }_{0} F_{2}\left(1,2 ;|\alpha|^{2}\right)}} \sum_{n=0}^{\infty} \frac{\alpha^{n}}{n ! \sqrt{(n+1) !}}\left|\psi_{n+1}\right\rangle,
$$

where ${ }_{0} F_{2}\left(1,2 ;|\alpha|^{2}\right)$ is a generalized hypergeometric function defined by [31]

$$
{ }_{0} F_{2}(x, y ; z)=\sum_{n=0}^{\infty} \frac{\Gamma(x) \Gamma(y)}{\Gamma(x+n) \Gamma(y+n)} \frac{z^{n}}{n !} .
$$

We now discuss its relationship with some states of the oscillator, using the identity techniques presented in Sec.2.3. In fact, equation (3.11) can be rewritten as

$$
\begin{aligned}
|\alpha\rangle & =b^{\dagger} \frac{1}{\sqrt{{ }_{0} F_{2}\left(1,2,|\alpha|^{2}\right)}} \sum_{n=0}^{\infty} \frac{\alpha^{n}}{n !(n+1) \sqrt{n !}}|n\rangle \\
& =b^{\dagger} \frac{1}{\sqrt{{ }_{0} F_{2}\left(1,2 ;|\alpha|^{2}\right)}} \sum_{n=0}^{\infty} \frac{\alpha^{n}}{n !(n+1) !}\left(a^{\dagger}\right)^{n}|0\rangle \\
& =b^{\dagger} \frac{1}{\sqrt{{ }_{0} F_{2}\left(1,2 ;|\alpha|^{2}\right)}} \exp \left(\frac{\alpha}{N+1} a^{\dagger}\right)|0\rangle .
\end{aligned}
$$


It is easy to see that

$$
\left[(N+2) a, \frac{1}{N+1} a^{\dagger}\right]=1
$$

therefore, the (unnormalized) state

$$
\frac{1}{\sqrt{{ }_{0} F_{2}\left(1,2 ;|\alpha|^{2}\right)}} \exp \left(\frac{\alpha}{N+1} a^{\dagger}\right)|0\rangle
$$

is the eigenstate of the operator $(N+2) a$, a density-dependent annihilation operator of the oscillator. Therefore the coherent state can be obtained by applying the operator $b^{\dagger}$ to the eigenstate of the operator $(N+2) a$.

On the other hand, what is the state obtained by applying the operator $b$ to $|\alpha\rangle$ ? We in fact have

$$
b|\alpha\rangle=\frac{1}{\sqrt{{ }_{0} F_{2}\left(1,2 ;|\alpha|^{2}\right)}} \sum_{n=0}^{\infty} \frac{\alpha^{n}}{n ! n !}\left(a^{\dagger}\right)^{n}|0\rangle=\frac{1}{\sqrt{{ }_{0} F_{2}\left(1,2 ;|\alpha|^{2}\right)}} \exp \left(\frac{\alpha}{N} a^{\dagger}\right)|0\rangle,
$$

which is the eigenstate of the density-dependent annihilation operator $(N+1) a$ of the oscillator. So the coherent states of the ISO are connected with the eigenstates of the densitydependent annihilation operators $(N+2) a$ and $(N+1) a$, in terms of the transformation $b^{\dagger}$ and $b$.

Then we consider the squeezed vacuum defined by

$$
\left(\mu A+\nu A^{\dagger}\right)|v\rangle=0
$$

where the complex numbers $\mu$ and $\nu$ satisfy $|\nu / \mu|<1$. Taking $|v\rangle=\sum_{n=0}^{\infty} C_{n}\left|\psi_{n}\right\rangle$, and inserting it into the equation (3.17), we obtain

$$
C_{2 k}=0, \quad C_{2 k+1}=z^{k}\left(\frac{(2 k-1) ! !}{(2 k) ! !(2 k+1)}\right)^{\frac{1}{2}} C_{1}, \quad z \equiv-\frac{\nu}{\mu},
$$

Then we have

$$
|v\rangle=C_{1} \sum_{k=0}^{\infty} z^{k}\left(\frac{(2 k-1) ! !}{(2 k) ! !(2 k+1)}\right)^{\frac{1}{2}}\left|\psi_{2 k+1}\right\rangle=b^{\dagger} C_{1} \sum_{k=0}^{\infty} \frac{z^{k}}{(2 k+1) k !}\left(\frac{a^{\dagger 2}}{2}\right)^{k}|0\rangle .
$$

The state

$$
C_{1} \sum_{k=0}^{\infty} \frac{1}{2 k+1} \frac{z^{k}}{k !}\left(\frac{a^{\dagger 2}}{2}\right)^{k}|0\rangle
$$

cannot be written in the form of an exponential operator acting on the vacuum state $|0\rangle$. However, this state can be transformed to an exponential state by the action of $b$. It is easy to see that

$$
b|v\rangle=C_{1} \sum_{k=0}^{\infty} \frac{1}{k !}\left(z \frac{a^{\dagger 2}}{2}\right)^{k}|0\rangle .
$$


After normalization this state is nothing but the squeezed vacuum of the oscillator

$$
b|v\rangle=S(z)\left|\psi_{0}\right\rangle \equiv \exp \left(z \frac{a^{\dagger 2}}{2}-z^{*} \frac{a^{2}}{2}\right)|0\rangle .
$$

Therefore the squeezed vacuum of ISOS is closely related to that of the oscillator through the transformation $b$.

\section{$4 q-\mathrm{GDO}$ and PB phase operator}

The purpose of this section is twofold: (1) we construct the new creation and annihilation operators related with the $\mathrm{PB}$ phase operator theory which form a closed associative algebra (some $q$-deformed GDO) and degenerate to those of the ordinary oscillator in certain limit; (2) we present a formalism to define algebraically the hermitian phase operator from the viewpoint of the cyclic representations of some $q$-GDO.

\section{1 q-GDO and its cyclic representation}

The GDO $\mathcal{B}$ in general does not admit the cyclic representation. To connect the GDO with the hermitian phase operator, we have to look for the GDO which has finite dimensional cyclic representations in the same sense as in the $q$-oscillator [26].

Define the algebra $\mathcal{B}_{q}$ as an associative algebra with generators $A^{\dagger}, A, q^{\mathcal{N}}$ and 1 subject to the relations

$$
q^{\mathcal{N}} A=q^{-1} A q^{\mathcal{N}}, \quad q^{\mathcal{N}} A^{\dagger}=q A^{\dagger} q^{\mathcal{N}}, \quad A A^{\dagger}=\mathcal{F}\left(q^{\mathcal{N}+1}\right), \quad A^{\dagger} A=\mathcal{F}\left(q^{\mathcal{N}}\right)
$$

where the hermitian non-negative function $\mathcal{F}$ is again called the structure function. This algebra can be obviously viewed as a subalgebra of the algebra $\mathcal{B}$ by identifying $\mathcal{F}\left(q^{\mathcal{N}}\right) \equiv$ $F(\mathcal{N})$.

The algebra $\mathcal{B}_{q}$ admits the Fock-like representation for any $q$, if the condition $\mathcal{F}(1)=0$ $\Leftrightarrow F(0)=0$ is satisfied. However, when $q$ is the $(S+1)$-th root of unity, some other types of representations are possible. Here we are interested in the cyclic representations, for which the condition $\mathcal{F}(1)=0$ is not imposed. We first prove that, if $q$ is the $(S+1)$-th root of unity, the elements $A^{S+1},\left(A^{\dagger}\right)^{S+1}$ and $\left(q^{\mathcal{N}}\right)^{S+1}$ are all the central elements of the algebra $\mathcal{B}_{q}$ . This can be shown from (4.1) and the following relations

$$
\begin{aligned}
& {\left[A^{S+1}, A^{\dagger}\right]=A^{S}\left(\mathcal{F}\left(q^{\mathcal{N}+1}\right)-\mathcal{F}\left(q^{\mathcal{N}-S}\right)\right)=0,} \\
& {\left[A,\left(A^{\dagger}\right)^{S+1}\right]=\left(\mathcal{F}\left(q^{\mathcal{N}+1}\right)-\mathcal{F}\left(q^{\mathcal{N}-S}\right)\right)\left(A^{\dagger}\right)^{S}=0,}
\end{aligned}
$$


since $q^{\mathcal{N}+1}=q^{\mathcal{N}-S+(S+1)}=q^{\mathcal{N}-S}$.

Now let us construct the explicit cyclic representation of $q$-GDO when $q^{S+1}=1$. Let $\mathcal{H}_{S}$ be a vector space with an orthonormal basis

$$
\mathcal{H}_{S+1}:\{|k\rangle \mid k=0,1,2, \cdots, S\} .
$$

Define the action of $\mathcal{B}_{q}$ on $\mathcal{H}_{S}$ as

$$
\begin{array}{ll}
A|k\rangle=\sqrt{\mathcal{F}\left(q^{k+\eta}\right)}|k-1\rangle, \quad k \neq 0, & A|0\rangle=\xi^{-1} \sqrt{\mathcal{F}\left(q^{\eta}\right)}|S\rangle, \quad \xi \neq 0, \\
A^{\dagger}|k\rangle=\sqrt{\mathcal{F}\left(q^{k+\eta+1}\right)}|k+1\rangle, \quad k \neq S, \quad & A^{\dagger}|S\rangle=\xi \sqrt{\mathcal{F}\left(q^{\eta}\right)}|0\rangle, \\
q^{\mathcal{N}}|k\rangle=q^{k+\eta}|k\rangle, &
\end{array}
$$

where $\eta$ is an extra real parameter (which may depend on $S$ ) [27] and $\xi \neq 0$ is a complex constant. One can directly verify that Eqs.(4.4) define an $(S+1)$-dimensional cyclic representation of $\mathcal{B}_{q}$ if

$$
\mathcal{F}\left(q^{\eta+k}\right) \neq 0 \quad \text { for } \quad k=0,1, \cdots, S .
$$

In this representation the central elements take

$$
\begin{aligned}
& \left(q^{\mathcal{N}}\right)^{S+1}=q^{\eta(S+1)}, \quad\left(A^{\dagger}\right)^{S+1}=\xi \sqrt{\mathcal{F}\left(q^{\eta}\right) \mathcal{F}\left(q^{\eta+1}\right) \cdots \mathcal{F}\left(q^{\eta+S}\right)}, \\
& A^{S+1}=\xi^{-1} \sqrt{\mathcal{F}\left(q^{\eta}\right) \mathcal{F}\left(q^{\eta+1}\right) \cdots \mathcal{F}\left(q^{\eta+S}\right)},
\end{aligned}
$$

which are non-vanishing constants.

It should be remarked that the naive $q$-oscillator with $q^{S+1}=1$, namely

$$
\mathcal{F}\left(q^{\mathcal{N}}\right)=[\mathcal{N}]=\left(q^{\mathcal{N}}-q^{-\mathcal{N}}\right) /\left(q-q^{-1}\right)
$$

fails to provide a cyclic representation simply because $\mathcal{F}\left(q^{\mathcal{N}}\right)$ takes negative as well as positive values. Some admissible choices are:

$$
\begin{aligned}
& \mathcal{F}\left(q^{\mathcal{N}}\right)=|[\mathcal{N}]|=\left|\left(q^{\mathcal{N}}-q^{-\mathcal{N}}\right) /\left(q-q^{-1}\right)\right|, \\
& \mathcal{F}\left(q^{\mathcal{N}}\right)=\left|\left(q^{\mathcal{N}}-q^{-\mathcal{N}}\right) /\left(q-q^{-1}\right)+K(S)\right|,
\end{aligned}
$$

where $K(S)$ is real. Let us call them positive ' $q$-oscillators'. For the case (4.8), the condition $|[k+\eta]| \neq 0$ is satisfied if $\eta$ is not an integer. Then (4.4) defines a cyclic representation. For the case of (4.9) we can also choose $\eta$ and $K(S)$ to have cyclic representations by satisfying (4.5). For example,

$$
0<|K(S)|<\frac{1}{2} \quad \text { and } \quad \eta \equiv 0 .
$$

We will return to these examples in connection with the hermitian phase operator. 
Another well known type of $q$-deformed oscillator ( $q$ complex)

$$
\mathcal{F}\left(q^{\mathcal{N}}\right)=\frac{1-q^{\mathcal{N}}}{1-q}
$$

or equivalently,

$$
A A^{\dagger}-q A^{\dagger} A=1
$$

is not admissible as a $q$-GDO. It is obvious that the hermiticity condition of $\mathcal{F}$ is not satisfied.

Another example of the $q-\mathrm{GDO}$ is the dynamical symmetry algebra of the hamiltonian system with self-similar potentials [28]. In this system the symmetry algebra is ( $q$ : real)

$$
L B^{\dagger}=q^{2} B^{\dagger} L, \quad L B=q^{-2} B L, \quad B^{\dagger} B=\prod_{n=0}^{M}\left(L+\omega_{n}\right), \quad B B^{\dagger}=\prod_{n=0}^{M}\left(q^{2} L+\omega_{n}\right),
$$

where $L$ is the Hamiltonian, $\omega_{n}$ are some real and positive constants and $M$ is a positive integer. It can be rewritten in the form (4.1) by identification

$$
L \longrightarrow\left(q^{\mathcal{N}}\right)^{2}, \quad B^{\dagger} \longrightarrow A^{\dagger}, \quad B \longrightarrow A
$$

Next we shall prove that the $q$-GDO can also be realized in terms of the PB phase operators in quantum optics and on the other hand it can be used to define the hermitian phase operator.

\subsection{Creation and annihilation operators of PB phase operator the- ory}

Let us begin with PB's theory of hermitian phase operator. The PB phase operator is defined in an $(S+1)$-dimensional space $\mathcal{H}_{S}$ spanned by the number basis $|n\rangle, n=0,1, \cdots, S$, with the inner product $\langle m \mid n\rangle=\delta_{m n}$. Define the phase states $\left|\theta_{m}\right\rangle, m=0,1, \cdots, S$,

$$
\left|\theta_{m}\right\rangle=\frac{1}{\sqrt{S+1}} \sum_{n=0}^{S} \exp \left(i n \theta_{m}\right)|n\rangle,
$$

where $\theta_{m}=\theta_{0}+\frac{2 \pi m}{S+1}$ and $\theta_{0}$ are real constants. Hereafter we write $\exp \left(i n \theta_{m}\right)$ as

$$
\exp \left(i n \theta_{m}\right)=\exp \left(i n \theta_{0}\right) q^{m n}
$$

where $q$ is the deformation parameter

$$
q=\exp \left(\frac{2 \pi}{S+1} i\right)
$$

satisfying $q^{S+1}=1$. From the orthonormality of the number states $\langle m \mid n\rangle=\delta_{m n}$ it is easy to prove that of the phase states $\left\langle\theta_{m} \mid \theta_{n}\right\rangle=\delta_{m n}$. We can express the number states in terms of the phase states

$$
|n\rangle=\frac{1}{\sqrt{S+1}} \sum_{m=0}^{S} \exp \left(-i n \theta_{m}\right)\left|\theta_{m}\right\rangle
$$


The $P B$ phase operator is defined as

$$
\hat{\Phi}_{\theta}=\sum_{m=0}^{S} \theta_{m}\left|\theta_{m}\right\rangle\left\langle\theta_{m}\left|; \quad \hat{\Phi}_{\theta}\right| \theta_{m}\right\rangle=\theta_{m}\left|\theta_{m}\right\rangle .
$$

A representation of the exponential $\mathrm{PB}$ phase operator $\mathrm{e}^{i \hat{\Phi}_{\theta}}$ on $|n\rangle$ is obtained as

$$
\begin{aligned}
& \mathrm{e}^{i \hat{\Phi}_{\theta}}|n\rangle=|n-1\rangle, \quad n \neq 0, \\
& \mathrm{e}^{i \hat{\Phi}_{\theta}}|0\rangle=\exp \left\{i(S+1) \theta_{0}\right\}|S\rangle, \\
& \mathrm{e}^{-i \hat{\Phi}_{\theta}}|n\rangle=|n+1\rangle, \quad n \neq S, \\
& \mathrm{e}^{-i \hat{\Phi}_{\theta}}|S\rangle=\exp \left\{-i(S+1) \theta_{0}\right\}|0\rangle .
\end{aligned}
$$

At this stage, Pegg and Barnett defined the creation and the annihilation operators

$$
a_{\mathrm{PB}}^{\dagger}=\sqrt{\hat{N}} \mathrm{e}^{-i \hat{\Phi}_{\theta}}, \quad a_{\mathrm{PB}}=\mathrm{e}^{i \hat{\Phi}_{\theta}} \sqrt{\hat{N}}
$$

where

$$
\hat{N}=\sum_{n=0}^{S} n|n\rangle\langle n|
$$

Then $a_{\mathrm{PB}}^{\dagger}$ and $a_{\mathrm{PB}}$ satisfy the so-called truncated oscillator commutation relation

$$
\left[a_{\mathrm{PB}}, a_{\mathrm{PB}}^{\dagger}\right]=1-(S+1)|S\rangle\langle S|
$$

which they claim to degenerate to that of the ordinary oscillator

$$
\left\langle p\left|\left[a_{\mathrm{PB}}, a_{\mathrm{PB}}^{\dagger}\right]\right| p\right\rangle_{S \rightarrow \infty}=1
$$

on the "physical states" $|p\rangle$ (for example, on the coherent states of the single mode electromagnetic field) in the limit $S \rightarrow \infty$. We note that the truncated oscillator does not form a closed algebra and that the operator relations do not simply reduce to those of the ordinary oscillator in the limit $S \rightarrow \infty$.

Here we shall define new creation and annihilation operators, which form a closed algebra (some $q$-GDO introduced in Sec.4.1) and degenerate to the usual oscillator in the limit $S \rightarrow \infty$. For this purpose, let us define

$$
A^{\dagger}=\sqrt{\mathcal{F}\left(q^{\mathcal{N}}\right)} e^{-i \hat{\Phi}_{\theta}}, \quad A=e^{i \hat{\Phi}_{\theta}} \sqrt{\mathcal{F}\left(q^{\mathcal{N}}\right)}, \quad q^{\mathcal{N}}=q^{\hat{N}+\eta}=e^{i \frac{2 \pi \eta}{S+1}} e^{i \frac{2 \pi}{S+1} \hat{N}}
$$

where the operator $e^{i \frac{2 \pi}{S+1} \hat{N}}$ is nothing but the phase shift operator

$$
e^{i \frac{2 \pi}{S+1} \hat{N}}\left|\theta_{m}\right\rangle=\left|\theta_{m}+\frac{2 \pi}{S+1}\right\rangle \equiv\left|\theta_{m+1}\right\rangle
$$


and the parameter $\eta$ and the function $\mathcal{F}$ will be specified later. It is easy to see that Eqs.4.25) lead to the following relations

$$
\begin{aligned}
& A^{\dagger}|n\rangle=\sqrt{\mathcal{F}\left(q^{n+\eta+1}\right)}|n+1\rangle, \quad n \neq S, \\
& A^{\dagger}|S\rangle=\exp \left\{-i(S+1) \theta_{0}\right\} \sqrt{\mathcal{F}\left(q^{\eta}\right)}|0\rangle, \\
& A|n\rangle=\sqrt{\mathcal{F}\left(q^{n+\eta}\right)}|n-1\rangle, \quad n \neq 0, \\
& A|0\rangle=\sqrt{\mathcal{F}\left(q^{\eta}\right)} \exp \left\{i(S+1) \theta_{0}\right\}|S\rangle, \\
& q^{\mathcal{N}}|n\rangle=q^{n+\eta}|n\rangle,
\end{aligned}
$$

which are just the representation (4.4) of the $q$-GDO with $\xi=\exp \left\{-i(S+1) \theta_{0}\right\}$. Therefore the operators defined in (4.25) generate a $q$-GDO.

Now we consider the constraints on the parameter $\eta$ or $K(S)$ in the function $\mathcal{F}$. The following conditions should be satisfied:

1. The function $\mathcal{F}$ must be hermitian and non-negative due to the same properties of the operator $A^{\dagger} A$.

2. Choose $\eta$ such that the representation (4.27) is a cyclic representation.

3. In the classical limit $S \rightarrow \infty(q \rightarrow 1)$ or zero deformation, the operators $A^{\dagger}, A$ should tend to the creation and annihilation operators of the ordinary oscillator.

The condition for the cyclic representation is equivalent to the condition that the operator $\mathcal{F}\left(q^{\mathcal{N}}\right)$ has the inverse which is necessary in order to define the PB phase operator. For simplicity and concreteness, let us discuss the positive ' $q$-oscillators' (4.8),(4.9). As mentioned above the condition for non-vanishing central elements (4.5) can be easily satisfied for (4.8) case if $\eta(S)$ is not an integer. As for the other example (4.9), the same condition is satisfied for example by

$$
\eta \equiv 0, \quad 0<|K(S)|<\frac{1}{2}
$$

In either case, the algebra of $A$ and $A^{\dagger}$ degenerate to that of the ordinary oscillator (Weyl algebra) provided

$$
\lim _{S \rightarrow \infty} \eta(S)=0, \quad \text { or } \quad \lim _{S \rightarrow \infty} K(S)=0 .
$$

These can be achieved, for example, $\eta(S)=K(S)=\frac{1}{S+1}$.

We would like to remark that Ellinas 25] studied the phase operator from the regular representation of the naive $q$-oscillator with $q^{S+1}=1$. As remarked in the previous subsection, the naive $q$-oscillator (4.7) with $q$ root of unity does not possess an admissible algebraic 
structure to connect with the hermitian phase operator. Moreover, in the regular representation characterized by the condition $A|0\rangle=A^{\dagger}|S\rangle=0$, both of the operators $A$ and $A^{\dagger}$ have a zero mode. Therefore the polar decomposition for them does not exist and the hermiticity of the phase operator, if any, is not guaranteed.

\subsection{Algebraic definition of the hermitian phase operator}

Now we turn to the study of the hermitian phase operator from the viewpoint of the cyclic representations of $q$-GDO. For concreteness, we consider the positive ' $q$-oscillator' (4.8) only. For the other choices of $\mathcal{F}$ like (4.9) the discussion is essentially the same. We start with the cyclic representation (4.4) of positive ' $q$-oscillator' on the space $\mathcal{H}_{S}$ with the inner product $\langle m \mid n\rangle=\delta_{m n}$. Define the exponential phase operator $\mathrm{e}^{i \hat{\Phi}_{\theta}}$ and $\mathrm{e}^{-i \hat{\Phi}_{\theta}}$ by the relation (4.25). Since we have chosen $\eta$ such that $\sqrt{|[\eta+k]|} \neq 0(k \in\{0,1, \cdots, S\})$ in the cyclic representation $(4.25)$, the operator $\sqrt{|[\mathcal{N}]|}$ (4.4) has the inverse. Therefore in the cyclic representation (4.4) the operators $e^{ \pm i \hat{\Phi}_{\theta}}$ are well defined uniquely:

$$
e^{i \hat{\Phi}_{\theta}}=\{|[\mathcal{N}+1]|\}^{-\frac{1}{2}} A, \quad e^{-i \hat{\Phi}_{\theta}}=\{|[\mathcal{N}]|\}^{-\frac{1}{2}} A^{\dagger}
$$

Inserting (4.30) into (4.4), we find the action of $e^{ \pm i \hat{\Phi}_{\theta}}$ on $\mathcal{H}_{S}$

$$
\begin{aligned}
& e^{i \hat{\Phi}_{\theta}}|k\rangle=|k-1\rangle, \quad k \neq 0, \quad e^{i \hat{\Phi}_{\theta}}|0\rangle=\xi^{-1}|S\rangle, \\
& e^{-i \hat{\Phi}_{\theta}}|k\rangle=|k+1\rangle, \quad k \neq S, \quad e^{-i \hat{\Phi}_{\theta}}|S\rangle=\xi|0\rangle, \\
& q^{\mathcal{N}}|k\rangle=q^{k+\eta}|k\rangle .
\end{aligned}
$$

Choosing $\xi=e^{-i \theta_{0}(S+1)}$, we exactly reproduce PB's exponential phase operator (4.27).

It is not convenient to derive the action of phase operator $\hat{\Phi}_{\theta}$ itself on the number states. To evaluate the phase operator $\hat{\Phi}_{\theta}$ itself, we have to look for a basis on which $\mathrm{e}^{i \hat{\Phi}_{\theta}}$ is diagonal. To this end we evaluate the eigenstates of $\mathrm{e}^{i \hat{\Phi}_{\theta}}$

$$
\mathrm{e}^{i \hat{\Phi}_{\theta}}|z\rangle=z|z\rangle
$$

Suppose that $|z\rangle=\sum_{n=0}^{S} C_{n}|n\rangle$, where $C_{n}$ is coefficient to be determined. Then inserting it into the Eqs.(4.32) we obtain $S+1$ distinct eigenvalues

$$
z_{m}=\exp \left(i \theta_{0}\right) \exp \left(\frac{2 \pi m i}{S+1}\right) \equiv \exp \left(i \theta_{m}\right), \quad m=0,1, \cdots, S
$$

where $\theta_{m}$ is same as in Eq.(4.16). Then the corresponding eigenstates are

$$
\left|\theta_{m}\right\rangle \equiv\left|z_{m}\right\rangle=C_{0} \sum_{n=0}^{S} \exp \left(i \theta_{m} n\right)|n\rangle,
$$


and their inner product

$$
\left\langle\theta_{m} \mid \theta_{n}\right\rangle=\left|C_{0}\right|^{2}(S+1) \delta_{m n}
$$

Requiring that the states $\left|\theta_{m}\right\rangle$ are normalized, the constant $C_{0}$ is fixed as $\frac{1}{\sqrt{S+1}}$. In comparison with PB's theory, these eigenstates form the phase states. On the phase states the eigenvalue equation (4.32) becomes

$$
\mathrm{e}^{i \hat{\Phi}_{\theta}}\left|\theta_{m}\right\rangle=\exp \left(i \theta_{m}\right)\left|\theta_{m}\right\rangle
$$

from which we can define the hermitian phase operator $\hat{\Phi}_{\theta}$ as follows

$$
\hat{\Phi}_{\theta}\left|\theta_{m}\right\rangle=\theta_{m}\left|\theta_{m}\right\rangle
$$

So, this approach exactly recovers PB's theory. It should be noted, however, that all the eigenvalues could be shifted by an integer multiple of $2 \pi$, which is natural for a phase. In other words it can be absorbed by the redefinition of $\theta_{0}$.

\section{Conclusion}

In this paper we have studied the GDO and some of its properties, namely, the multiphoton realizations, the ladder-operator coherent and squeezed vacuum states. The coherent displacement-operator $D(\alpha)$ and the squeeze operator $S(z)$ are explicitly constructed and expressed in the exponential form. For the ordinary oscillator, we know that the state $D(\alpha) S(z)|0\rangle$

$$
|\alpha, z\rangle \equiv D(\alpha) S(z)|0\rangle=C_{0} e^{\alpha a^{\dagger}} e^{z a^{\dagger 2}}|0\rangle \stackrel{\text { normalization }}{=} e^{\alpha a^{\dagger}-\alpha^{*} a} e^{z a^{\dagger 2}-z^{*} a^{2}}|0\rangle,
$$

is just the squeezed state, which is also the eigenstate of $\mu a+\nu a^{\dagger}$. However, for the general case (the GDO), the state $D(\alpha) S(z) \| 0\rangle$ is not the squeezed state equivalent to the ladderoperator definition, namely, it is not the eigenstate of $\mu A+\nu A^{\dagger}$. The coherent displacementoperator $D(\alpha)$ is a good operator in the sense that it enjoys the following property

$$
D(-\alpha) A D(\alpha)=A+\alpha
$$

However, the squeeze operator $S(z)$ does not keep the Bogoliubov transformation, namely

$$
S^{-1}(z) A S(z) \neq \mu A+\nu A^{\dagger}
$$

This is why the state $D(\alpha) S(z)|0\rangle$ is not the eigenstate of $\mu A+\nu A^{\dagger}$, as is argued in [30]. However, we can expect that the states $D(\alpha) S(z) \| 0\rangle$ and $S(z) D(\alpha) \| 0\rangle$ are important quantum states in quantum optics and it is a good challenge to study their nonclassical properties. 
We have pointed out that a realistic physical system, the ISOS (isospectral oscillator system), has GDO as its symmetry algebra. Its coherent and squeezed vacuum states are studied in some detail and that they are compared and related with those of the ordinary oscillator.

To connect the GDO with the hermitian phase operator, we have introduced a new algebra, the $q$-GDO, which is a subalgebra of GDO depending on a complex parameter $q$. It has cyclic representations when $q$ is a root of unity. This approach has two remarkable advantages: (1) The phase operator of the Pegg-Barnett's theory can be constructed from the $q$-GDO purely algebraically; (2) The $q$-GDO with $q^{S+1}=1$ provides a finite dimensional space to define the phase operator and the cyclic representations ensure the hermiticity of the phase operator in contrast with the regular representation case.

\section{Acknowledgments}

We thank D. Fairlie, P. Kulish, M.Pillin and C.Zachos for useful comments. H. C.Fu is grateful to Japan Society for Promotion of Science (JSPS) for the fellowship. He is also supported in part by the National Science Foundation of China.

\section{Appendix A: Multimode GDO}

The formalism in Sec.2.1 can be easily generalized to the multimode case. For simplicity we consider only the two-mode case. Generalization to three-mode and further is straightforward. Consider the two-mode photon field described by two independent modes

$$
\left[a, a^{\dagger}\right]=1, \quad\left[b, b^{\dagger}\right]=1
$$

and an arbitrary two-mode multiphoton annihilation oscillator

$$
A=f\left(N_{1}, N_{2}\right) a^{m} b^{n}
$$

where $N_{1}=a^{\dagger} a, N_{2}=b^{\dagger} b, f$ is an arbitrary function with $f\left(n_{1}, n_{2}\right) \neq 0$ for $n_{1}, n_{2}$ nonnegative integers. Note that $f$ is not necessarily factorized as $f\left(N_{1}, N_{2}\right)=f_{1}\left(N_{1}\right) f_{2}\left(N_{2}\right)$. It is easy to see $(i=1,2)$

$$
A A^{\dagger}=F\left(\mathcal{N}_{1}+1, \mathcal{N}_{2}+1\right), \quad A^{\dagger} A=F\left(\mathcal{N}_{1}, \mathcal{N}_{2}\right), \quad\left[\mathcal{N}_{i}, A^{\dagger}\right]=A^{\dagger}, \quad\left[\mathcal{N}_{i}, A\right]=-A,
$$

where

$$
\mathcal{N}_{1} \equiv \frac{1}{m}\left(N_{1}-i\right), \quad \mathcal{N}_{2} \equiv \frac{1}{n}\left(N_{1}-j\right), \quad(0 \leq i \leq m-1,0 \leq j \leq n-1),
$$




$$
\begin{aligned}
F\left(\mathcal{N}_{1}+1, \mathcal{N}_{2}+1\right) \equiv\left(N_{1}+1\right) \cdots\left(N_{1}+m\right)\left(N_{2}+1\right) \cdots\left(N_{2}+n\right) f\left(N_{1}, N_{2}\right) f^{*}\left(N_{1}, N_{2}\right) \\
\equiv \\
\left(m \mathcal{N}_{1}+i+1\right) \cdots\left(m \mathcal{N}_{1}+i+m\right)\left(n \mathcal{N}_{2}+j+1\right) \cdots \\
\quad\left(n \mathcal{N}_{2}+j+n\right) f\left(\mathcal{N}_{1}, \mathcal{N}_{2}\right) f^{*}\left(\mathcal{N}_{1}, \mathcal{N}_{2}\right) .
\end{aligned}
$$

This algebra is defined in a subspace $\bar{S}_{i j}$ of the sector $S_{i j}$ spanned by $(k=0,1,2, \cdots)$

$$
\| k\rangle \equiv \frac{1}{\sqrt{F(k, k) !}}\left(A^{\dagger}\right)^{k}|i, j\rangle \propto|k m+i, k n+j\rangle .
$$

The representation on $\bar{S}_{i j}$ is

$$
\left.\left.\left.\left.\left.\left.\left.A^{\dagger} \| k\right\rangle=\sqrt{F(k, k)} \| k+1\right\rangle, \quad A \| k\right\rangle=\sqrt{F(k, k)} \| k-1\right\rangle, \quad \mathcal{N}_{1} \| k\right\rangle=\mathcal{N}_{2} \| k\right\rangle=k \| k\right\rangle .
$$

We consider the eigenvalue equation

$$
\left(\mu A+\nu A^{\dagger}\right)|\beta\rangle=\beta|\beta\rangle
$$

These states are degenerate. The degeneracy can be lifted by assuming that the $(m+n)$ photons are either created or annihilated together. This means the following conservation law

$$
\left(\mathcal{N}_{1}-\mathcal{N}_{2}\right)|\beta\rangle=0
$$

In the representation (A.6) the condition is fulfilled automatically.

Identifying $F(k, k)$ here with $F(k)$ in section 2, the representation (A.6) takes the same form as (2.15). So, formally, the squeezed states can be investigated in the same manner as those in section 2.2 .

\section{References}

[1] Drinfeld VG 1986 in Proceedings of the International Congress of Mathematicians ed. Gleason A M (American Mathematical Society, Providence, RI) 798

[2] Jimbo M 1986 Lett. Math. Phys. 11247

[3] See, for example, Rampacher H, Stumpf H and Wagner F 1965 Fortschritte der Physik 13385

[4] Arik M and Coon D D 1976 J. Math. Phys. 17524

[5] Kuryshkin V 1980 Annales de la Fondation Louis de Broglie 5111 
[6] Biedenharn LC 1989 J. Phys. A 22 L873; Macfarlane A J 1989 J.Phys. A 22 4581; Sun CP and Fu H C 1989 J. Phys. A 22 L983

[7] Bayen F, Flato M, Fronsdal C, Lichnerowicz A and Sternheimer D 1978 Ann. Phys. 111 $61 ; 111111$

[8] Bonatsos D, Daskaloyannis C 1993 Phys. Lett. B. 307100

[9] Bonatsos D, Daskaloyannis C and Kolokotronis P 1995 Mod. Phys. Lett. A 102197

[10] Karassiov V P 1994 J. Phys. A: Math. Phys. 27 153; Karassiov V P and Klimov A B 1994 Phys. Lett. A. 191117

[11] Mielnik B 1984 J. Math. Phys. 253387

[12] NietoL M 1984 Phys. Lett. B 145 208; Cao X C 1991 J. Phys. A 24 L1155

[13] Shanta P, ChaturvediS, Srinivasan V and Jagannathan R 1994 J. Phys. A 276433

[14] Brandt R, Greenberg OW 1969 J. Math. Phys. 10 1168; Katriel J, Solomon A I, D’Ariano G and RasettiM 1986 Phys.Rev.D 34 2332; 1987 Phys. Rev. D 341248 ; D’Ariano G, Morosi S, Rasetti M, Katriel J and Solomon A I 1987 Phys. Rev. D 36 2399; Vourdas A 1993 J. Math. Phys. 34 1223; 1994352687

[15] Chang Z 1992 J. Math. Phys. 333172

[16] Katriel J and Solomon A I 1991 J. Phys. A 242093

[17] Atakishiev NM $\quad$ and $\quad$ SuslovSK $\quad 1990 \quad$ Theor. Math. Phys. 85 1055; 1991 Theor. Math. Phys. 87 442; Atakishiev N M, Frank A and Wolf K B 1994 J. Math. Phys. 353253

[18] Song X C 1990 J. Phys. A 23 821; Kulish P P and Damaskinsky E V 1990 J. Phys. A 23 L415; Chaichian M and Kulish P P 1990 Phys. Lett. B234 72; Celeghini E, Rasetti M and Vitiello G 1991 Phys. Rev. Lett. 66 2056; Fairlie D B and Nuyts J 1993 J. Math. Phys. 34 4441; Zachos C 1992 Contemp. Math. 134 351; Pillin M 1995 Preprint YITP/U-95-32, q-alg/9508014

[19] Fernández C D J, Hussin V and Nieto L M 1995 J. Phys. A 273547

[20] Dirac P A M 1927 Proc. R. Soc. London 114243 
[21] Quantum Physics and Phase Dependent Measurements 1993 ed. Schleich W P and Barnett SM Physica Scripta T48

[22] Susskind M and Glogower J 1964 Physics 149

[23] Pegg D T and BarnettSM 1988 Europhys. Lett. 6 6; 1989 Phys. Rev. A 39 1665; Barnet S M and Pegg D T 1989 J. Mod. Phys. 367

[24] Ellinas D 1991 J. Math. Phys. 32 135; 1991 J. Mod. Phys. 382393

[25] Ellinas D 1992 Phys. Rev. A 453358

[26] Fu H C and Ge ML 1992 J. Math. Phys. 33427

[27] Odaka K, Kishi T and Kamefuchi S 1991 J. Phys. A 24 L591

[28] SkorikS and SpiridonovV 1993 Schrödinger operators with q-ladder symmetry algebra, in: Third International Workshop on squeezed states and Uncertainty relations, ed. Han D, Kim YS, Rubin NH and Zachary WW (NASA Conference Publications 3270); Spiridonov V 1993 Comm. Theor. Phy. 2

[29] Chaturvedi S, Kapoor A K, Sandhya R, Srinivasan V and Simon R 1991 Phys. Rev. A 43 4555

[30] Nieto M M and Truax D R 1995 Preprint LA-UR-95-1996, quant-ph/9506025

[31] Bateman H 1953 Higher Transcendental Functions, Vol.1, ed. Erdelyi A (New York: McCraw Hill) 